\title{
Delta Fractional Sturm-Liouville Problems: From Discrete to Continuous
}

\author{
Ramazan Ozarslan ${ }^{1 *}$ Erdal Bas ${ }^{2}$ \\ ${ }^{1,2}$ Department of Mathematics, Science Faculty, Firat University, 23119 Elazig, Turkey
}

\begin{abstract}
In this study, we consider delta fractional Sturm-Liouville (DFSL) initial value problems in the sense of delta Caputo and delta Riemann-Liouville (R-L) operators. One of the properties of delta fractional difference operators which makes it different from nabla counterpart is to shift its domain. This feature makes it more complex than the nabla fractional operator. We obtain sum representation of solutions for DFSL initial value problems with the help of $\mathcal{Z}$ - transformation. Moreover, we get analytical solutions of homogeneous DFSL problem within RiemannLiouville (R-L) and Caputo sense, discrete Sturm-Liouville (DSL) problem, continuous fractional Sturm-Liouville (FSL) problem in the sense of R-L and Caputo operators, and continuous Sturm-Liouville (SL) differential problem. From this point of view, we compare all the solutions with each other. Consequently, we show that all results for these four eigenvalue problems are compatible with each other and approach to each other while the orders tends to one, i.e. $\Delta^{\mu}(\Delta x(t-\mu)) \cong D_{0^{+}}^{\mu}\left(x^{\prime}(t)\right) \cong \Delta^{2} x(n-1) \cong x^{\prime \prime}(t)=\lambda x(t), \mu \rightarrow 1$. We support our results comparatively by tables and simulations in detail.
\end{abstract}

Keywords: Delta fractional equations, Fractional differential equations, Sturm-Liouville, $\mathcal{Z}$ - Transform, Eigenfunctions, Eigenvalues.

MSC: Primary: 34A08, 39A70, 34B24 ; Secondary: 44A10.

\section{Introduction}

The idea of a fractional derivative was first proposed in 1695 on the question of L'Hospital about the meaning of the derivative $\frac{d^{n} y}{d x^{n}}$ while $n=\frac{1}{2}$ to Leibniz. Leibniz gave that answer, "It appears that one day useful consequences will be drawn from these paradoxes.". Thereafter, Leibniz and Bernoulli began to research the meaning of the derivative of $\frac{1}{2}$.th order of a power function by the help of the definition of generalized derivative. First known definitions of fractional derivatives are Riemann-Liouville and Caputo derivatives. In addition to these definitions, new fractional derivatives have been defined in the recent past. Some of them are Hilfer [1] in 2000, Hadamard [2] in 2001, Caputo-Fabrizio [3] in 2015, Atangana-Baleanu [4] in 2016, and Hilfer-Katugampola [5] in 2017.

Fractional difference operators were first given by Chapman in 1911 [6] for $\Delta^{s} a_{n}$ (for $s \in \mathbb{R}$ ). Various fractional difference operators were defined by Kuttner [7] in 1957, Diaz-Osler [8] in 1974, and Granger-R. Joyeux [9], Isaacs [10] in 1980, Hosking [11] in 1981, Miller-Ross [12] in 1988,

${ }^{*}$ Corresponding author. E-mail: ozarslanramazan@gmail.com (R. Ozarslan) 
and Hirota [13] in 2000. Anastassiou [14] defined the delta discrete fractional Caputo operator, and also defined nabla discrete fractional Caputo operator [15]. Abdeljawad [16] studied properties of the delta Caputo fractional difference operator in 2011, and also dual identities of the delta RiemannLiouville fractional difference operator [17] in 2013. Then, Abdeljawad [18] studied relation between delta and nabla discrete fractional Caputo operators. In fact, instead of the fractional expression, noninteger expression is more appropriate because we can take the $\sqrt{3} t h$ order derivative and the $\pi t h$ order difference of a function. However, we will continue to use "fractional" expression in a usual way.

Although the existence of considerable studies in the theoretical viewpoint of fractional differential equations [19-22], there has been no significant parallel development of fractional difference equations until very recently. In recent times, there has also been a huge increase in the theoretical viewpoint of discrete fractional equations. With this development, unexpected difficulties and technical complications emerged in fractional difference equations. One of these complications seems in the fractional Sturm-Liouville difference equations. Strictly speaking, fractional Sturm-Liouville differential equations is a relatively new subject, with a history dating back to the last 10 years, and improvement is still needed in its theory. In 2012, Klimek-Agrawal [23] analyzed the reality of eigenvalues and orthogonality of eigenfunctions for the regular fractional S-L problems, and Ciesielski et al. [24] investigated fractional S-L problems in a numerical way in 2017. In 2013, Bas and Metin [25, 26] studied spectral properties of the singular fractional S-L differential operator, as well as the self-adjointness of the operator, Dehghan-Mingarelli [27] studied the fractional S-L problems in 2017. In 2016, Almeida et al. [28] analyzed the variational properties of solutions by dealing with the fractional Sturm-Liouville difference equations with the Grünwald-Letnikov delta fractional difference operator. Following, some spectral properties of conformable Sturm-Liouville and nabla fractional Sturm-Liouville with R-L and Caputo-Fabrizio operator has been given by [29-31]. Recently, numerous studies about Atangana-Baleanu fractional derivative and its nabla discrete versions are done [32,33].

Mathematical modeling is needed for the study of physical events in the real world. Therefore, boundary value differential problems are needed to model problems in many areas of mathematical physics, science and engineering. While the solution of these problems can be expressed analytically until the 1830s, in 1836 Charles François Sturm and Joseph-Liouville made studies on the properties and behaviors of these solutions when the solutions could not be calculated analytically and they established the Sturm-Liouville theory.

Basic spectral properties such as operator's self-adjointness, orthogonality of eigenfunctions and reality of eigenvalues have an important place in Sturm-Liouville theory. Besides, the problem of finding the spectral data called the direct problem is the representation of the solutions, the finding of asymptotic formulas of eigenfunctions and eigenvalues, spectral data, spectral functions, scattering data, and spectral data such as norming constants.

If we mention in the physical applications of Sturm-Liouville problems, we can say about heat conduction problems, heat flow problems, acoustics, aerodynamics, elastic, electrodynamic, fluid dynamics, seismic wave propagation, heat transfer, ocean science, optics, petroleum engineering, quantum mechanics. Sturm considered the problem of heat conduction in a non-homogeneous fine wire, and in the 1880s Rayleigh and Kirchhoff analyzed the vibration problem and applied the same Sturm's theorems to boundary value problems.

In this study, Sturm-Liouville differential equations of integer and fractional order are discussed. The results of the solutions of fractional delta Riemann-Liouville and delta Caputo Sturm-Liouville difference problems are obtained and the solutions obtained with four different operators are compared with integer order delta difference, fractional order delta difference, integer order continuous and 
fractional continuous. However, solutions of delta Caputo fractional and Caputo fractional continuous Sturm-Liouville problems are compared, similarly solutions of delta Riemann-Liouville fractional and Riemann-Liouville fractional continuous Sturm-Liouville problems are compared and all results obtained in detail with simulations.

Therefore, the content of this study is quite popular and original. Thus, a strong basis was obtained in the classical subjects of fractional Sturm-Liouville difference problems, and at the same time the fractional differential-continuous differential was compared with the integer order difference and fractional difference versions, and the connection between of them was shown.

Delta fractional difference equations has been studied recently by Atıc1 et al. [34-36], Mozyrska et al. [37 38], Baleanu et al. [39], Abdeljawad [16]17], Mohan-Deekshitulu [40]. In 2012, Abdeljawad et al. [41] discussed nabla Caputo discrete Mittag-Leffler (M-L) functions for fractional linear difference systems. One of the properties of delta fractional difference operators which makes it different from nabla counterpart is to shift its domain. This feature makes it more complex than the nabla fractional difference. This difficulty will not be seen in the nabla fractional difference operator.

$\mathcal{Z}$ - transformation method dates back to 1730s. De Moivre [42] defined the generating function. This definition is very similar to the $\mathcal{Z}$ - transformation. Later, the definition of generating function was used more extensively in 1812 with Laplace [43]. Applications of $\mathcal{Z}$ - transform are seen in probability theory, system and control theory as a result of using digital computers in systems. The $\mathcal{Z}$ - transformation method can be seen as the difference counterpart of Laplace transform used in the calculation of differential equations. Bohner and Peterson [44] studied delta Laplace transformation in their study. The Delta Laplace transformation is equivalent to the $\mathcal{Z}$ - transformation under one transformation.

M-L function was first described by G. M. Mittag-Leffler [45] in 1903. In 2003, Nagai [46] first described the discrete M-L function. In 2007 Atıc1-Eloe [47] described the R-transformation for the solution of delta fractional difference equations. In 2011, Holm [48] described the delta Laplace transformation for delta fractional difference equations. In 2013, Abu-Saris and Al-Mdallal [49] used the $\mathcal{Z}$ - transform to investigate the stability conditions of the Caputo type systems. In 2014, Xiaoyan and Wei [50] described the Laplace transform and the discrete M-L function for the Caputo delta fractional difference equations. In 2015, Mozyrska and Wyrwas [51] used the classical $\mathcal{Z}-$ transform for delta fractional differential equation systems, and from there they defined the discrete $\mathrm{M}-$ $\mathrm{L}$ function and established a connection between the $\mathcal{Z}$ - transformation and discrete $\mathrm{M}-\mathrm{L}$ functions. In 2016, Wu et al. [52] obtained solutions of Caputo delta fractional difference equations and obtained numerical solutions with the approximate value of the M-L function. In 2018, Anh et al. [53] used the classical $\mathcal{Z}$ - transformation for delta fractional difference equation systems and, using the discrete $\mathrm{M}$ $\mathrm{L}$ function, described the method of variation of parameters for delta fractional differential equation systems. In our work, in terms of convenience, the method used in [53] is preferred for the delta fractional Sturm-Liouville equations.

\section{Mathematical Background}

\subsection{Fractional Differential Operators}

Definition 1. [20] $R-L$ fractional derivative is given as, $\alpha>0, \alpha \in \mathbb{R}$ and $n-1 \leq \alpha<n, n \in \mathbb{N}$,

$$
{ }_{a} D_{x}^{\alpha} f(x)=\frac{1}{\Gamma(n-\alpha)}\left(\frac{d}{d t}\right)^{n} \int_{a}^{x} f(t)(x-t)^{n-\alpha-1} d t .
$$


Definition 2. [20] Caputo fractional derivative is given as, $\alpha>0, \alpha \in \mathbb{R}$ and $n-1 \leq \alpha<n, n \in \mathbb{N}$,

$$
{ }_{a}^{C} D_{x}^{\alpha} f(t)=\frac{1}{\Gamma(n-\alpha)} \int_{a}^{x} \frac{d^{n}}{d t^{n}} f(t)(x-t)^{n-\alpha-1} d t .
$$

Definition 3. [20] 2-parameters $M-L$ function is defined for $z, \beta \in \mathbb{C}, \operatorname{Re}(\alpha)>0$

$$
E_{\alpha, \beta}(z)=\sum_{k=0}^{\infty} \frac{z^{k}}{\Gamma(\alpha k+\beta)} .
$$

Theorem 1. [20] The Laplace transform (LT) of 2-parameters Mittag-Leffler functions has the following property

$$
\mathcal{L}\left\{t^{\beta-1} E_{\alpha, \beta}\left(\lambda t^{\alpha}\right)\right\}(s)=\frac{s^{\alpha-\beta}}{s^{\alpha}-\lambda} .
$$

Property 2.1. [20] $f: \mathbb{N}_{a} \rightarrow \mathbb{R}, 0<\alpha<1$, the LT of Caputo fractional derivative operator is given as

$$
\mathcal{L}\left\{{ }^{C} D_{0^{+}}^{\alpha} f\right\}(s)=s^{\alpha} \mathcal{L}\{f\}(s)-s^{\alpha-1} f(0) .
$$

Property 2.2. [20] $f: \mathbb{N}_{a} \rightarrow \mathbb{R}, 0<\alpha<1$, the LT of $R-L$ fractional derivative operator is given as

$$
\mathcal{L}\left\{D_{0^{+}}^{\alpha} f\right\}(s)=s^{\alpha} \mathcal{L}\{f\}(s)-\left.I_{0^{+}}^{1-\alpha} f(t)\right|_{t=0},
$$

where $I_{0^{+}}^{\alpha}$ is Riemann-Liouville fractional integral.

Definition 4. $[54] n \in \mathbb{Z}^{+} \cup\{0\}$ falling factorial function is given as

$$
t^{\underline{n}}=t(t-1)(t-2) \ldots(t-n+1),
$$

here $t^{0}=1$.

Theorem 2. [55] $t, r \in \mathbb{R}^{+}$, generalized factorial function is given as

$$
t^{\underline{r}}=\frac{\Gamma(t+1)}{\Gamma(t-r+1)},
$$

here, $t$ and $r$ must be selected so that the gamma function is defined.

Now, let us give the relation between gamma function and falling factorial function:

$$
\left(\begin{array}{c}
t \\
r
\end{array}\right)=\frac{t^{\underline{r}}}{\Gamma(r+1)}
$$

\subsection{Delta Fractional Difference and Sum Operators}

Definition 5. 12 35] $x: \mathbb{N}_{a+\mu} \rightarrow \mathbb{R}, m-1<\mu<m, m \in \mathbb{Z}^{+}, \mu \in \mathbb{R}^{+}$, delta fractional sum operators are defined as follows:

$i$. Let $\mu>0, \mu$. order left defined delta fractional sum;

$$
\Delta_{a}^{-\mu} x(t)=\frac{1}{\Gamma(\mu)} \sum_{s=a}^{t-\mu}(t-\sigma(s)) \frac{\mu-1}{x} x(s), t \in \mathbb{N}_{a+\mu},
$$


ii. Let $\mu>0, \mu$. order right defined delta fractional sum;

$$
{ }_{b} \Delta^{-\mu} x(t)=\frac{1}{\Gamma(\mu)} \sum_{s=t+\mu}^{b}(s-\sigma(t)) \frac{\mu-1}{x} x(s), t \in{ }_{b-\mu} \mathbb{N} .
$$

Definition 6. 12,35$]$ Delta fractional difference operators are given as follows

i. Let $\mu>0$, $\mu$. order left defined Riemann-Liouville $(\boldsymbol{R}-\boldsymbol{L})$ delta fractional difference operator, $t \in \mathbb{N}_{a+n-\mu}$

$$
\Delta_{a}^{\mu} x(t)=\Delta^{n} \Delta_{a}^{-(n-\mu)} x(t)=\frac{\Delta^{n}}{\Gamma(n-\mu)} \sum_{s=a}^{t-n+\mu}(t-\sigma(s)) \frac{n-\mu-1}{n} x(s),
$$

ii. Let $\mu>0, \mu$. order right defined Riemann-Liouville $(\boldsymbol{R}-\mathbf{L})$ delta fractional difference operator; $t \in{ }_{b-n+\mu} \mathbb{N}$

$$
{ }_{b} \Delta^{\mu} x(t)=(-1)^{n} \nabla_{b}^{n} \Delta^{-(n-\mu)} x(t)=\frac{(-1)^{n} \nabla^{n}}{\Gamma(n-\mu)} \sum_{s=t+n-\mu}^{b}(s-\sigma(t)) \frac{n-\mu-1}{x} x(s) .
$$

Definition 7. $14,16,18] \mu>0, \mu \notin \mathbb{N}$, $\mu$. order delta Caputo fractional difference operators are given as follows:

i. $x$, is defined on $\mathbb{N}_{a}$, and let $\mu>0$, $\mu$. order left defined delta Caputo difference operator, $t \in$ $\mathbb{N}_{a+n-\mu}$,

$$
{ }^{C} \Delta_{a}^{\mu} x(t)=\Delta_{a}^{-(n-\mu)} \Delta^{n} x(t)=\frac{1}{\Gamma(n-\mu)} \sum_{s=a}^{t-n+\mu}(t-\sigma(s)) \frac{n-\mu-1}{n} \Delta^{n} x(s),
$$

ii. $x$, is defined on ${ }_{b} \mathbb{N}$, and let $\mu>0, \mu$. order right defined delta Caputo fractional difference operator; $t \in{ }_{b-n+\mu} \mathbb{N}$

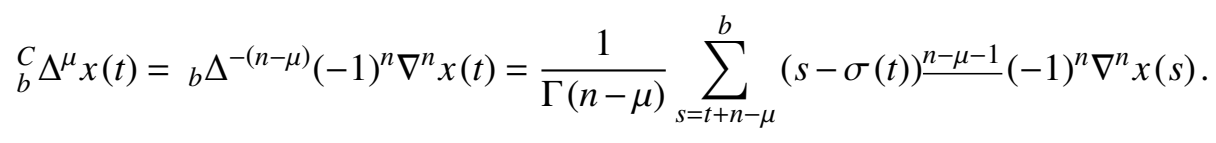

\subsection{Z-Transformation for Delta Fractional Difference Equations}

Definition 8. $[56] f: \mathbb{N}_{a} \rightarrow \mathbb{R}, z \in \mathbb{C}$, delta $\mathcal{Z}$ - transformation is given as follows, $|z|>R, R=$ $\limsup _{k \rightarrow \infty}|f(k)|^{1 / k}$,

$$
\mathcal{Z}[f](z)=\sum_{k=0}^{\infty} f(k) z^{-k}
$$

Lemma 2.1. $f: \mathbb{N}_{a-1} \rightarrow \mathbb{R}, z \in \mathbb{C}$, delta Z-transformation has the following property $|z|>R, R=$ $\lim \sup |f(k)|^{1 / k}$,

$$
\mathcal{Z}[f](z-1)=\frac{1}{z} \mathcal{Z}[f](z)+f(-1) .
$$


Definition 9. [56] (Delta convolution property) $f, g: \mathbb{N}_{a} \rightarrow \mathbb{R}$, the convolution of $f$ and $g$ functions is defined as

$$
(f * g)(n)=\sum_{k=0}^{t} f(n-k) g(k) .
$$

Theorem 3. [56] (Delta Convolution Theorem) $f, g: \mathbb{N} a \rightarrow \mathbb{R}$,

$$
\mathcal{Z}[f * g](n)=\mathcal{Z}[f] \mathcal{Z}[g](n) .
$$

Theorem 4. $[56]\left(\mathcal{Z}\right.$-transformation of nth order delta difference ) $f: \mathbb{N}_{a} \rightarrow \mathbb{R}$

$$
\mathcal{Z}\left[\Delta^{n} f\right](z)=(z-1)^{n} \mathcal{Z}[f](z)-z \sum_{k=0}^{n-1}(z-1)^{n-k-1} \Delta^{k} f(0) .
$$

Theorem 5. $[51,53] f: \mathbb{N}_{a} \rightarrow \mathbb{R}, 0<\mu<1, \mathcal{Z}$-transformations of Caputo delta and $R-L$ delta fractional difference operators are as following respectively:

$$
\begin{aligned}
\mathcal{Z}\left[{ }^{C} \Delta_{a}^{\mu} f\right](z+1-\mu) & =z\left(\frac{z}{z-1}\right)^{-\mu}\left[\mathcal{Z}[f](z)-\frac{z}{z-1} f(0)\right], n \in \mathbb{N}_{a+1-\mu}, \\
\mathcal{Z}\left[\Delta_{a}^{\mu} f\right](z+1-\mu) & =z\left(\frac{z}{z-1}\right)^{-\mu} \mathcal{Z}[f](z)-z f(0), n \in \mathbb{N}_{a+1-\mu} .
\end{aligned}
$$

Definition 10. 37,53$]$ (Delta discrete $M-L$ function) $\operatorname{Re}(\alpha)>0, \beta, t \in \mathbb{C}, A \in \mathbb{R}$ and $t \in \mathbb{N}_{a}$, delta discrete $M-L$ function is given as follows;

$$
\begin{gathered}
E_{(\alpha, \beta)}(A, n)=\sum_{k=0}^{\infty} A^{k}\left(\begin{array}{c}
n-k+k \alpha+\beta-1 \\
n-k
\end{array}\right), n \in \mathbb{Z}, \\
\mathcal{E}_{(\alpha, \beta)}(A, z)=\sum_{k=0}^{\infty} A^{k} \frac{(z+(k-1)(\mu-1))^{\alpha k+\beta}(z+k(\mu-1))^{\beta-1}}{\Gamma(\alpha k+\beta)}, z \in \mathbb{C} .
\end{gathered}
$$

There exists a relation between these two functions although these two functions are different from each other;

$$
E_{(\alpha, \alpha)}(A, n)=\mathcal{E}_{(\alpha, \alpha)}(A, n+1-\mu), n \in \mathbb{N} .
$$

Theorem 6. [53, 57] $A \in \mathbb{R}, x: \mathbb{N} \rightarrow \mathbb{R}, \mathcal{Z}$-transformation of delta discrete $M-L$ function is given as follows;

i. $\mathcal{Z}\left[E_{(\alpha, \beta)}(A,).\right](z)=\left(\frac{z}{z-1}\right)^{\beta}\left(1-\frac{1}{z}\left(\frac{z}{z-1}\right)^{\alpha} A\right)^{-1}$,

ii. $\mathcal{Z}\left[E_{(\alpha, \beta)}(A, .-1)\right](z)=\frac{1}{z}\left(\frac{z}{z-1}\right)^{\beta}\left(1-\frac{1}{z}\left(\frac{z}{z-1}\right)^{\alpha} A\right)^{-1}$,

iii. $\mathcal{Z}\left[E_{(\alpha, \beta)}(A, .-2)\right](z)=\frac{1}{z^{2}}\left(\frac{z}{z-1}\right)^{\beta}\left(1-\frac{1}{z}\left(\frac{z}{z-1}\right)^{\alpha} A\right)^{-1}$,

iv. $\mathcal{Z}\left[E_{(\alpha, \beta)}(A, .+1)\right](z)=z\left(\frac{z}{z-1}\right)^{\beta}\left(1-\frac{1}{z}\left(\frac{z}{z-1}\right)^{\alpha} A\right)^{-1}$,

where $|z|>1,|z-1|^{\alpha}|z|^{1-\alpha}>|A|$ and $\alpha \in(0,1]$. 


\section{Delta Fractional Sturm-Liouville Problems}

In this section, we consider delta fractional Sturm-Liouville (DFSL) equations in two different forms as;

1. (delta left) Caputo fractional difference operator,

$$
L_{1} x(t)={ }^{C} \Delta_{a}^{\mu}(\Delta x(t+1-\mu))+q(t) x(t)=\lambda x(t), 0<\mu<1,
$$

here $x: \mathbb{N} \rightarrow \mathbb{R}, q(t)$ is a real valued function reel, $\lambda$ is spectral parameter, $t \in \mathbb{N}_{a+1-\mu}^{b+1-\mu}, a, b$ is a finite integer.

2. (delta left) Riemann-Liouville (R-L) fractional difference operator,

$$
L_{2} x(t)=\Delta_{a}^{\mu}(\Delta x(t+1-\mu))+q(t) x(t)=\lambda x(t), 0<\mu<1,
$$

here $x: \mathbb{N} \rightarrow \mathbb{R}, q(t)$ is a real valued function reel, $\lambda$ is spectral parameter, $t \in \mathbb{N}_{a+1-\mu}^{b+1-\mu}, a, b$ is a finite integer.

\subsection{Representation of Solutions of Delta Fractional Sturm-Liouville Problems}

\subsubsection{Delta Fractional Sturm-Liouville Problem within Caputo Sense}

Theorem 7. Let us consider the following ivp

$$
\begin{gathered}
L_{1} x(t)={ }^{C} \Delta_{0}^{\mu}(\Delta x(t+1-\mu))+q(t) x(t)=\lambda x(t), 0<\mu<1, \\
x(0)=c_{1}, \Delta x(0)=c_{2},
\end{gathered}
$$

here $x: \mathbb{N} \rightarrow \mathbb{R}, t \in \mathbb{N}_{1-\mu}^{b+1-\mu}, b$ is a finite integer., $q(t)$ are defined on $[0,1-\mu]$ and real valued, $\lambda$ is a spectral parameter. Representation of solution of Caputo DFSL problem (21) - 22) is as follows

$$
\begin{aligned}
x(t)= & \frac{c_{1}}{2}\left[E_{\left(\frac{\mu+1}{2}, 1\right)}(\sqrt{\lambda}, t)+E_{\left(\frac{\mu+1}{2}, 1\right)}(-\sqrt{\lambda}, t)\right]+\frac{c_{2}}{2}\left[E_{\left(\frac{\mu+1}{2}, 2\right)}(\sqrt{\lambda}, t-1)+E_{\left(\frac{\mu+1}{2}, 2\right)}(-\sqrt{\lambda}, t-1)\right] \\
& -\frac{1}{2} \sum_{k=0}^{n}\left[E_{\left(\frac{\mu+1}{2}, \mu+1\right)}(\sqrt{\lambda}, t-k-2)+E_{\left(\frac{\mu+1}{2}, \mu+1\right)}(-\sqrt{\lambda}, t-k-2)\right] q(k) x(k),
\end{aligned}
$$

here $|t|>1,|t-1|^{\alpha}|t|^{1-\alpha}>|\lambda|$ due to Theorem 6 .

Proof. If we take $\mathcal{Z}$-transformation of both side of the equation 21) from Theorem 5, we have

$$
\begin{aligned}
\mathcal{Z}\left[{ }^{C} \Delta_{0}^{\mu}(\Delta x(.+1-\mu))\right](z)+\mathcal{Z}[q x](z) & =\lambda \mathcal{Z}[x](z), \\
z^{2}\left(\frac{z}{z-1}\right)^{-\mu-1} \mathcal{Z}[x](z)-z^{2}\left(\frac{z}{z-1}\right)^{-\mu} c_{1}-z\left(\frac{z}{z-1}\right)^{-\mu+1} c_{2} & =\lambda \mathcal{Z}[x](z)-\mathcal{Z}[q x](z) .
\end{aligned}
$$

From here

$$
\mathcal{Z}[x](z)=\frac{z^{2}\left(\frac{z}{z-1}\right)^{-\mu}}{z^{2}\left(\frac{z}{z-1}\right)^{-\mu-1}-\lambda} c_{1}+\frac{z\left(\frac{z}{z-1}\right)^{-\mu+1}}{z^{2}\left(\frac{z}{z-1}\right)^{-\mu-1}-\lambda} c_{2}-\frac{1}{z^{2}\left(\frac{z}{z-1}\right)^{-\mu-1}-\lambda} \mathcal{Z}[q x](z) .
$$


Now, let us take inverse $\mathcal{Z}$-transformation of both side of the equation (24). For this aim firstly let us make the terms in the rhs of the equation (24) suitable respectively to apply Theorem $6 i$.. For the first term at the rhs of the equation (24) from Theorem $6 i$;

$$
\begin{aligned}
\frac{z^{2}\left(\frac{z}{z-1}\right)^{-\mu}}{z^{2}\left(\frac{z}{z-1}\right)^{-\mu-1}-\lambda} & =\frac{1}{2} \frac{z}{z-1}\left(1-\frac{1}{z}\left(\frac{z}{z-1}\right)^{\frac{\mu+1}{2}} \sqrt{\lambda}\right)^{-1}+\frac{1}{2} \frac{z}{z-1}\left(1+\frac{1}{z}\left(\frac{z}{z-1}\right)^{\frac{\mu+1}{2}} \sqrt{\lambda}\right)^{-1}, \\
& =\frac{1}{2} E_{\left(\frac{\mu+1}{2}, 1\right)}(\sqrt{\lambda}, t)+\frac{1}{2} E_{\left(\frac{\mu+1}{2}, 1\right)}(-\sqrt{\lambda}, t) .
\end{aligned}
$$

For the second term at the rhs of the equation (24) from Theorem 6 ii.;

$$
\begin{aligned}
\frac{z\left(\frac{z}{z-1}\right)^{-\mu+1}}{z^{2}\left(\frac{z}{z-1}\right)^{-\mu-1}-\lambda} & =\frac{1}{2} \frac{1}{z}\left(\frac{z}{z-1}\right)^{2}\left(1-\frac{1}{z}\left(\frac{z}{z-1}\right)^{\frac{\mu+1}{2}} \sqrt{\lambda}\right)^{-1}+\frac{1}{2} \frac{1}{z}\left(\frac{z}{z-1}\right)^{2}\left(1+\frac{1}{z}\left(\frac{z}{z-1}\right)^{\frac{\mu+1}{2}} \sqrt{\lambda}\right)^{-1}, \\
& =\frac{1}{2} E_{\left(\frac{\mu+1}{2}, 2\right)}(\sqrt{\lambda}, t-1)+\frac{1}{2} E_{\left(\frac{\mu+1}{2}, 2\right)}(-\sqrt{\lambda}, t-1)
\end{aligned}
$$

For the third term at the rhs of the equation (24) from Theorem 6 iii.;

$$
\begin{aligned}
\frac{1}{z^{2}\left(\frac{z}{z-1}\right)^{-\mu-1}-\lambda} & =\frac{1}{2} \frac{1}{z^{2}}\left(\frac{z}{z-1}\right)^{\mu+1}\left(1-\frac{1}{z}\left(\frac{z}{z-1}\right)^{\frac{\mu+1}{2}} \sqrt{\lambda}\right)^{-1}+\frac{1}{2} \frac{1}{z^{2}}\left(\frac{z}{z-1}\right)^{\mu+1}\left(1+\frac{1}{z}\left(\frac{z}{z-1}\right)^{\frac{\mu+1}{2}} \sqrt{\lambda}\right)^{-1}, \\
& =\frac{1}{2} E_{\left(\frac{\mu+1}{2}, \mu+1\right)}(\sqrt{\lambda}, t-2)+\frac{1}{2} E_{\left(\frac{\mu+1}{2}, \mu+1\right)}(-\sqrt{\lambda}, t-2) .
\end{aligned}
$$

Also, if we use convolution property in Theorem 3, then we have

$$
\begin{aligned}
& \mathcal{Z}^{-1}\left[z \longmapsto \frac{1}{z^{2}\left(\frac{z}{z-1}\right)^{-\mu-1}-\lambda} \mathcal{Z}[q x](z)\right](t)= \\
& \sum_{k=0}^{n}\left[E_{\left(\frac{\mu+1}{2}, \mu+1\right)}(\sqrt{\lambda}, t-k-2)+E_{\left(\frac{\mu+1}{2}, \mu+1\right)}(-\sqrt{\lambda}, t-k-2)\right] q(k) x(k) .
\end{aligned}
$$

Finally, sum representation of solution of the problem $[21-22$ is found as follows

$$
\begin{aligned}
x(t)= & \frac{c_{1}}{2}\left[E_{\left(\frac{\mu+1}{2}\right)}(\sqrt{\lambda}, t)+E_{\left(\frac{\mu+1}{2}\right)}(-\sqrt{\lambda}, t)\right]+\frac{c_{2}}{2}\left[E_{\left(\frac{\mu+1}{2}, 2\right)}(\sqrt{\lambda}, t-1)+E_{\left(\frac{\mu+1}{2}, 2\right)}(-\sqrt{\lambda}, t-1)\right] \\
& -\frac{1}{2} \sum_{k=0}^{n}\left[E_{\left(\frac{\mu+1}{2}, \mu+1\right)}(\sqrt{\lambda}, t-k-2)+E_{\left(\frac{\mu+1}{2}, \mu+1\right)}(-\sqrt{\lambda}, t-k-2)\right] q(k) x(k) .
\end{aligned}
$$

Hence, the proof is completed.

\subsubsection{Delta Fractional Sturm-Liouville Problem within Riemann-Liouville Sense}

Theorem 8. Let us consider the following ivp

$$
\begin{gathered}
L_{2} x(t)=\Delta_{0}^{\mu}(\Delta x(t+1-\mu))+q(t) x(t)=\lambda x(t), 0<\mu<1 \\
x(0)=c_{3}, \Delta x(0)=c_{4},
\end{gathered}
$$


here $x: \mathbb{N} \rightarrow \mathbb{R}, t \in \mathbb{N}_{1-\mu}^{b+1-\mu}$, $b$ is a finite integer., $q(t)$ are defined on $[0,1-\mu]$ and real valued, $\lambda$ is a spectral parameter. Representation of solution of Riemann-Liouville DFSL problem (25) - 26) is as follows

$$
\begin{aligned}
x(t)= & \frac{c_{3}}{2}\left[E_{\left(\frac{\mu+1}{2}, 1\right)}(\sqrt{\lambda}, t)+E_{\left(\frac{\mu+1}{2}, 1\right)}(-\sqrt{\lambda}, t)\right]+\frac{c_{4}}{2}\left[E_{\left(\frac{\mu+1}{2}, \mu+1\right)}(\sqrt{\lambda}, t-1)+E_{\left(\frac{\mu+1}{2}, \mu+1\right)}(-\sqrt{\lambda}, t-1)\right] \\
& -\frac{1}{2} \sum_{k=0}^{n}\left[E_{\left(\frac{\mu+1}{2}, \mu+1\right)}(\sqrt{\lambda}, t-k-2)+E_{\left(\frac{\mu+1}{2}, \mu+1\right)}(-\sqrt{\lambda}, t-k-2)\right] q(k) x(k)
\end{aligned}
$$

here $|t|>1,|t-1|^{\alpha}|t|^{1-\alpha}>|\lambda|$ due to Theorem 6 .

Proof. If we take $\mathcal{Z}$-transformation of both side of the equation 25) from Theorem 5, we have

$$
\begin{aligned}
\mathcal{Z}\left[\Delta_{0}^{\mu}(\Delta x(.+1-\mu))\right](z)+\mathcal{Z}[q x](z) & =\lambda \mathcal{Z}[x](z), \\
z^{2}\left(\frac{z}{z-1}\right)^{-\mu-1} \mathcal{Z}[x](z)-z^{2}\left(\frac{z}{z-1}\right)^{-\mu} c_{3}-z c_{4} & =\lambda \mathcal{Z}[x](z)-\mathcal{Z}[q x](z),
\end{aligned}
$$

From here

$$
\mathcal{Z}[x](z)=\frac{z^{2}\left(\frac{z}{z-1}\right)^{-\mu}}{z^{2}\left(\frac{z}{z-1}\right)^{-\mu-1}-\lambda} c_{3}+\frac{z}{z^{2}\left(\frac{z}{z-1}\right)^{-\mu-1}-\lambda} c_{4}-\frac{1}{z^{2}\left(\frac{z}{z-1}\right)^{-\mu-1}-\lambda} \mathcal{Z}[q x](z) .
$$

Now, let us take inverse $\mathcal{Z}$-transformation of both side of the equation (28). For this aim firstly let us make the terms in the rhs of the equation (28) suitable respectively to apply Theorem $6 i$.

For the first term at the rhs of the equation (28) from Theorem $6 i$;

$$
\begin{aligned}
\frac{z^{2}\left(\frac{z}{z-1}\right)^{-\mu}}{z^{2}\left(\frac{z}{z-1}\right)^{-\mu-1}-\lambda} & =\frac{1}{2} \frac{z}{z-1}\left(1-\frac{1}{z}\left(\frac{z}{z-1}\right)^{\frac{\mu+1}{2}} \sqrt{\lambda}\right)^{-1}+\frac{1}{2} \frac{z}{z-1}\left(1+\frac{1}{z}\left(\frac{z}{z-1}\right)^{\frac{\mu+1}{2}} \sqrt{\lambda}\right)^{-1}, \\
& =\frac{1}{2} E_{\left(\frac{\mu+1}{2}, 1\right)}(\sqrt{\lambda}, t)+\frac{1}{2} E_{\left(\frac{\mu+1}{2}, 1\right)}(-\sqrt{\lambda}, t) .
\end{aligned}
$$

For the second term at the rhs of the equation 28 from Theorem 6 ii.;

$$
\begin{aligned}
\frac{z}{z^{2}\left(\frac{z}{z-1}\right)^{-\mu-1}-\lambda} & =\frac{1}{2} \frac{1}{z}\left(\frac{z}{z-1}\right)^{\mu+1}\left(1-\frac{1}{z}\left(\frac{z}{z-1}\right)^{\frac{\mu+1}{2}} \sqrt{\lambda}\right)^{-1}+\frac{1}{2} \frac{1}{z}\left(\frac{z}{z-1}\right)^{\mu+1}\left(1+\frac{1}{z}\left(\frac{z}{z-1}\right)^{\frac{\mu+1}{2}} \sqrt{\lambda}\right)^{-1}, \\
& =\frac{1}{2} E_{\left(\frac{\mu+1}{2}, \mu+1\right)}(\sqrt{\lambda}, t-1)+\frac{1}{2} E_{\left(\frac{\mu+1}{2}, \mu+1\right)}(-\sqrt{\lambda}, t-1) .
\end{aligned}
$$

For the third term at the rhs of the equation (28) from Theorem 6 iii.;

$$
\begin{aligned}
\frac{1}{z^{2}\left(\frac{z}{z-1}\right)^{-\mu-1}-\lambda} & =\frac{1}{2} \frac{1}{z^{2}}\left(\frac{z}{z-1}\right)^{\mu+1}\left(1-\frac{1}{z}\left(\frac{z}{z-1}\right)^{\frac{\mu+1}{2}} \sqrt{\lambda}\right)^{-1}+\frac{1}{2} \frac{1}{z^{2}}\left(\frac{z}{z-1}\right)^{\mu+1}\left(1+\frac{1}{z}\left(\frac{z}{z-1}\right)^{\frac{\mu+1}{2}} \sqrt{\lambda}\right)^{-1}, \\
& =\frac{1}{2} E_{\left(\frac{\mu+1}{2}, \mu+1\right)}(\sqrt{\lambda}, t-2)+\frac{1}{2} E_{\left(\frac{\mu+1}{2}, \mu+1\right)}(-\sqrt{\lambda}, t-2) .
\end{aligned}
$$


Also, if we use convolution property in Theorem 3, then we have

$$
\begin{aligned}
& \mathcal{Z}^{-1}\left[z \longmapsto \frac{1}{z^{2}\left(\frac{z}{z-1}\right)^{-\mu-1}-\lambda} \mathcal{Z}[q x](z)\right](t)= \\
& \sum_{k=0}^{n}\left[E_{\left(\frac{\mu+1}{2}, \mu+1\right)}(\sqrt{\lambda}, t-k-2)+E_{\left(\frac{\mu+1}{2}, \mu+1\right)}(-\sqrt{\lambda}, t-k-2)\right] q(k) x(k) .
\end{aligned}
$$

Finally, representation of solution of the problem $25-26$

$$
\begin{aligned}
x(t)= & \frac{c_{3}}{2}\left[E_{\left(\frac{\mu+1}{2}\right)}(\sqrt{\lambda}, t)+E_{\left(\frac{\mu+1}{2}\right)}(-\sqrt{\lambda}, t)\right]+\frac{c_{4}}{2}\left[E_{\left(\frac{\mu+1}{2}, \mu+1\right)}(\sqrt{\lambda}, t-1)+E_{\left(\frac{\mu+1}{2}, \mu+1\right)}(-\sqrt{\lambda}, t-1)\right] \\
& -\frac{1}{2} \sum_{k=0}^{n}\left[E_{\left(\frac{\mu+1}{2}, \mu+1\right)}(\sqrt{\lambda}, t-k-2)+E_{\left(\frac{\mu+1}{2}, \mu+1\right)}(-\sqrt{\lambda}, t-k-2)\right] q(k) x(k) .
\end{aligned}
$$

Hence, the proof is completed.

\subsection{Comparison of Delta Fractional and Delta Integer Order Sturm-Liouville Prob- lems with Fractional Continuous and Integer Order Continuous Sturm-Liouville Problems}

In this section, we obtain analytical solutions of delta fractional Sturm-Liouville (DFSL), delta integer order Sturm-Liouville (DSL), fractional Sturm-Liouville (FSL) and Sturm-Liouville differential (SL) problems while taking $q(t)=0$, and we compare all results obtained with figures and tables in detail.

\section{Analytical Solution of Caputo DFSL Problem}

DFSL problem:

$$
\begin{gathered}
{ }^{C} \Delta_{0}^{\mu}(\Delta x(t-\mu))=\lambda x(t), \\
x(-1)=1, \Delta x(-1)=1,
\end{gathered}
$$

here $x: \mathbb{N} \rightarrow \mathbb{R}, t \in \mathbb{N}_{-\mu}^{b-\mu}, b$ is a finite integer, and its analytic solution is as follows

$$
x(t)=-E_{(\mu+1,0)}(\lambda, t+1)+E_{(\mu+1,1)}(\lambda, t+1)+E_{(\mu+1,2)}(\lambda, t) .
$$

\section{Analytical Solution of DSL Problem}

DSL problem:

$$
\begin{gathered}
\Delta^{2} x(t-1)=\lambda x(t), \\
x(-1)=1, \Delta x(-1)=1,
\end{gathered}
$$

here $t \in \mathbb{N}_{-1}^{b-1}, b$ is a finite integer, and its analytic solution is as follows

$$
\begin{aligned}
x(t) & =\left(\frac{1+\lambda+\sqrt{\lambda(\lambda+4)}}{\sqrt{\lambda(\lambda+4)}}\right)\left(\frac{2+\lambda+\sqrt{\lambda(\lambda+4)}}{2}\right)^{t} \\
& +\left(\frac{1-\lambda+\sqrt{\lambda(\lambda+4)}}{\sqrt{\lambda(\lambda+4)}}\right)\left(\frac{2+\lambda-\sqrt{\lambda(\lambda+4)}}{2}\right)^{t} .
\end{aligned}
$$




\section{Analytical Solution of Caputo FSL Problem}

FSL problem:

$$
\begin{gathered}
{ }^{C} D_{0^{+}}^{\mu}\left(x^{\prime}(t)\right)=\lambda x(t), \\
x(0)=1, x^{\prime}(0)=1,
\end{gathered}
$$

$t \in[0, b]$ and its analytic solution is as follows

$$
x(t)=E_{\mu+1}\left(\lambda t^{\mu+1}\right)+t E_{\mu, 2}\left(\lambda t^{\mu+1}\right) .
$$

\section{Analytical Solution of Sturm-Liouville Differential Problem}

(SL) problem:

$$
\begin{gathered}
x^{\prime \prime}(t)=\lambda x(t), \\
x(0)=1, x^{\prime}(0)=1,
\end{gathered}
$$

$t \in[0, b]$ and its analytic solution is as follows

$$
\begin{gathered}
x(t)=\frac{1+\sqrt{\lambda}}{2 \sqrt{\lambda}} e^{\sqrt{\lambda} t}-\frac{1-\sqrt{\lambda}}{2 \sqrt{\lambda}} e^{-\sqrt{\lambda} t}, \\
x(t)=\cosh \sqrt{\lambda} t+\frac{\sinh \sqrt{\lambda} t}{\sqrt{\lambda}} .
\end{gathered}
$$

Where the domain and range of function $x(t)$ and $\mathrm{M}-\mathrm{L}$ functions must be selected to be defined. We can state that the solution of (35) - 36) FSL problem can be obtained by taking $\mu \rightarrow 1$ in the solution of (38) - 39) $S L$ problem and similarly, the solution of 29) - 30) DFSL problem can be obtained by taking $\mu \rightarrow 1$ in the solution of $D S L$ (34) problem.

\subsection{Visual Results}

Firstly, we compare the solution of the problems (29) - (30) DFSL ve (32) - (33) DSL and we show that while the order $\mu \rightarrow 1$ the solution of DFSL problem (31) approaches to the solution of DSL problem (34) in figure 1 . Let $\lambda=-0.01$.

Secondly, we compare the solutions of DFSL, DSL, FSL and SL problems At first view, we observe the solution of DSL and SL problems almost match in any order $\mu$, and we observe the solutions of DFSL and FSL problem almost match in any order $\mu$ in figures 2(a) 2(1). However, we observe that all of the solutions of DFSL, DSL, FSL and SL problems almost coincide to each other as $\mu=1$ in figures $2(\mathrm{~m}) 2(\mathrm{o})$, Let $\lambda=-0.01$. 


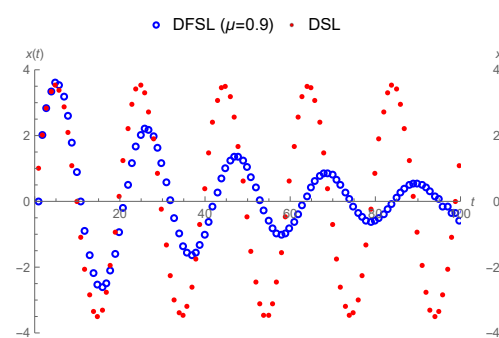

(a)
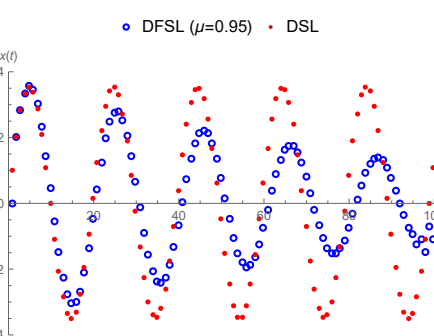

(b)

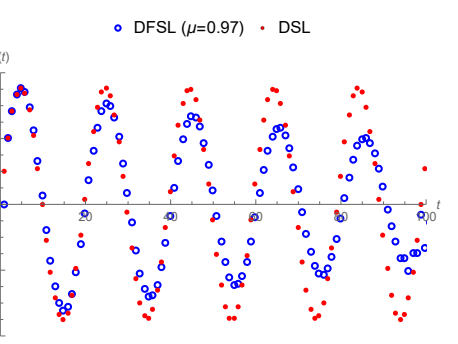

(c)

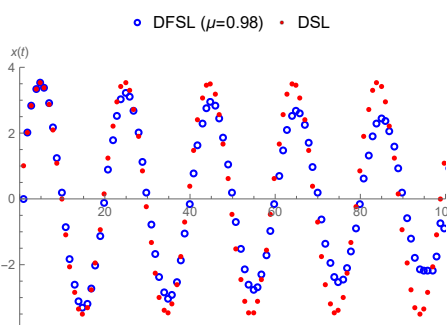

(d)

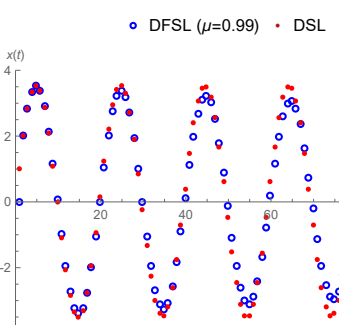

(e)

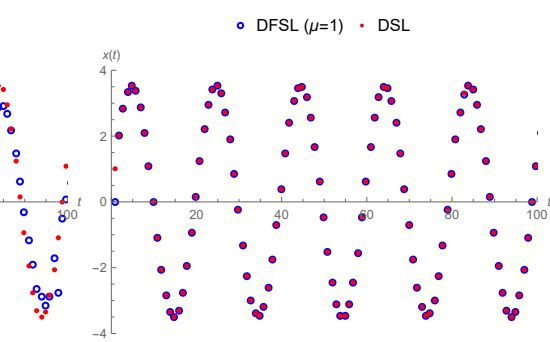

(f)

Figure 1

Thirdly, we compare the solution of DFSL problem (31) with different orders, potential functions and eigenvalues in the figures 6.3.22 6.3.25.

\section{Analytical Solution of Delta Riemann-Liouville Fractional Sturm-Liouville Problem} DFSL problem:

$$
\begin{gathered}
\Delta^{\mu}(\Delta x(t-\mu))=\lambda x(t), \\
x(-1)=1, \Delta x(-1)=1,
\end{gathered}
$$

here $x: \mathbb{N} \rightarrow \mathbb{R}, t \in \mathbb{N}_{-\mu}^{b-\mu}, b$ is a finite integer, and its analytic solution is as follows

$$
x(t)=-E_{(\mu+1,0)}(\lambda, t+1)+E_{(\mu+1,1)}(\lambda, t+1)+E_{(\mu+1, \mu+1)}(\lambda, t) .
$$

\section{Analytical Solution of Riemann-Liouville Fractional Sturm-Liouville Problem} FSL problem

$$
\begin{gathered}
D_{0^{+}}^{\mu}\left(x^{\prime}(t)\right)=\lambda x(t), \\
x(0)=1,\left.I_{0^{+}}^{1-\mu} x^{\prime}(t)\right|_{t=0}=1,
\end{gathered}
$$

$t \in[0, b]$ and its analytic solution is as follows

$$
x(t)=E_{\mu+1}\left(\lambda t^{\mu+1}\right)+t^{\mu+1} E_{\mu+1, \mu+1}\left(\lambda t^{\mu+1}\right) .
$$

Now, let us compare Caputo and Riemann-Liouville forms of these results obtained with figures visually. 


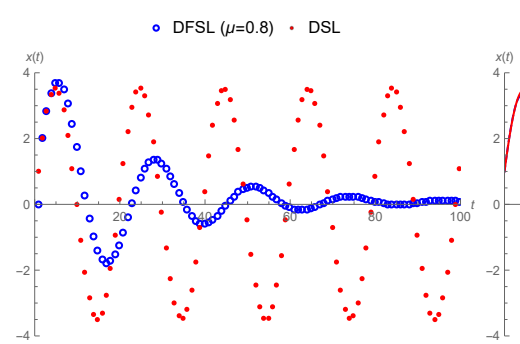

(a)

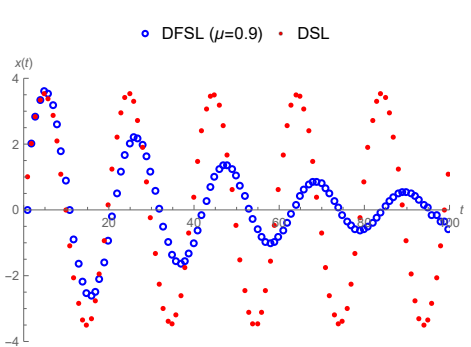

(d)

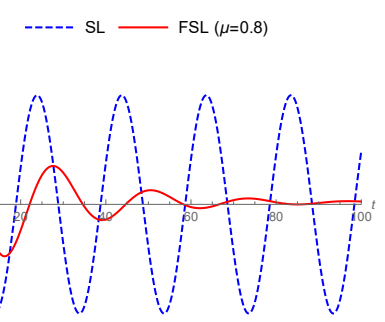

(b)

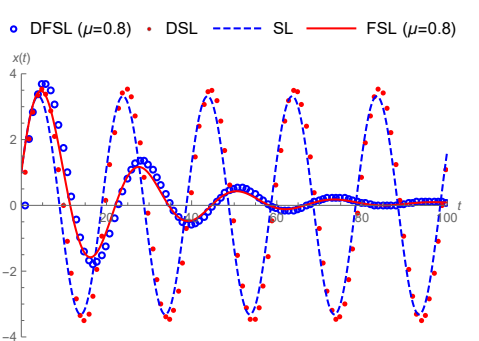

(c)

$$
\text { ---- SL — FSL }(\mu=0.9) \quad \text { ODFSL }(\mu=0.9) \cdot \text { DSL ----- SL } \longrightarrow \text { FSL }(\mu=0.9)
$$

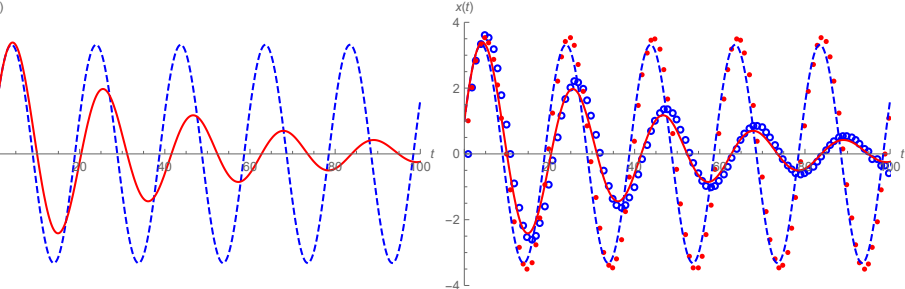

(e)

(f)

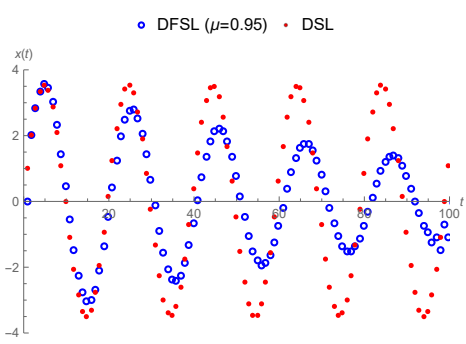

(g)
----- SL — FSL $(\mu=0.95)$

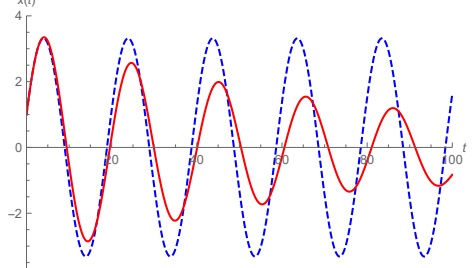

(h)

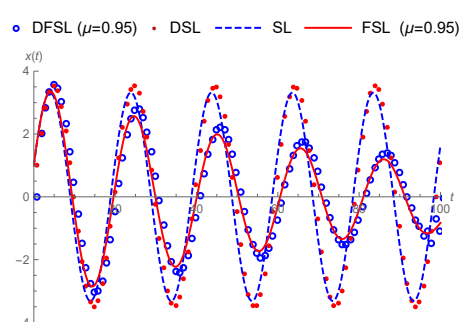

(i)

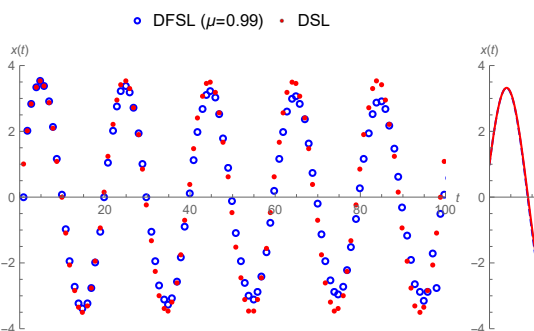

(j)

----- SL — FSL $(\mu=0.99)$ - DFSL $(\mu=0.99)$ • DSL ----- SL — FSL $(\mu=0.99)$

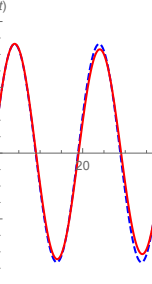

(k)

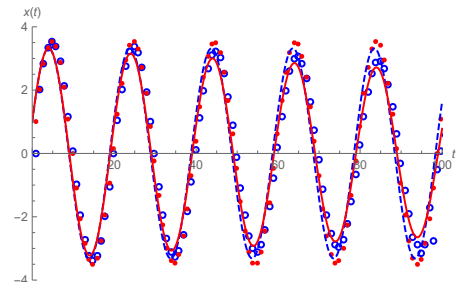

(1)

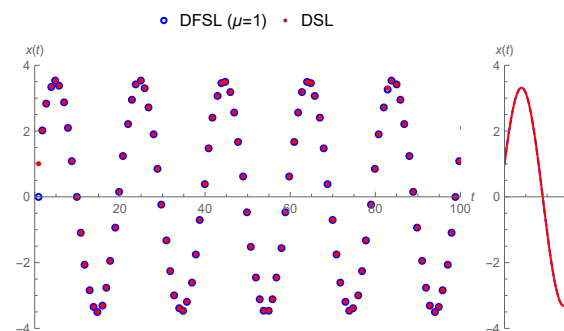

(m)
----- SL — FSL $(\mu=1)$

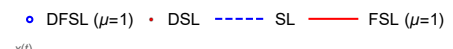

- DFSL $(\mu=1) \cdot \operatorname{DSL}-----\mathrm{SL}-\mathrm{FSL}(\mu=1)$

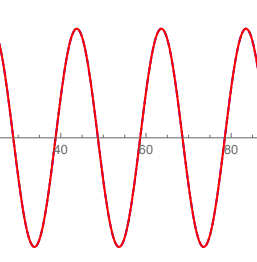

(n)

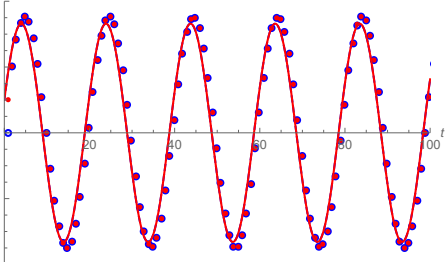

(o)

Figure 2 


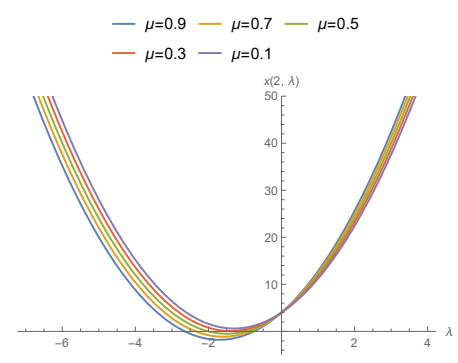

Figure 6.3.22 $q(t)=0$

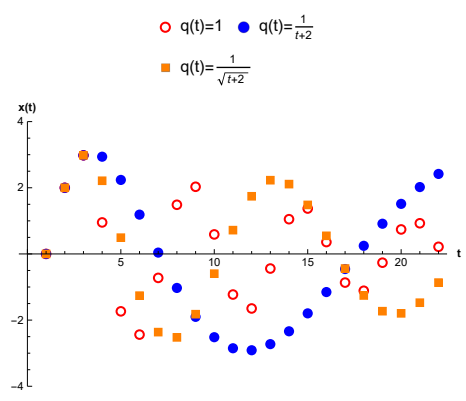

Figure 6.3.24 $\lambda=-0.01, \mu=0.9$

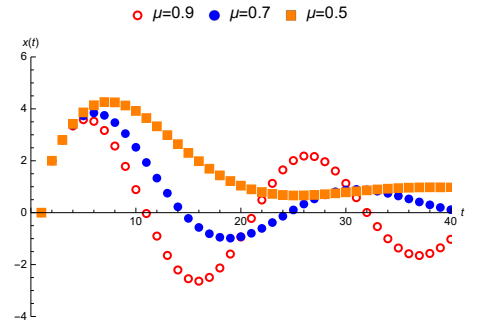

Figure 6.3.23 $\lambda=-0.01, q(t)=0$

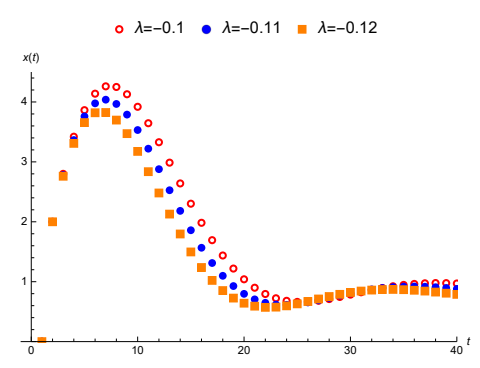

Figure 6.3.25 $q(t)=0, \mu=0.5$

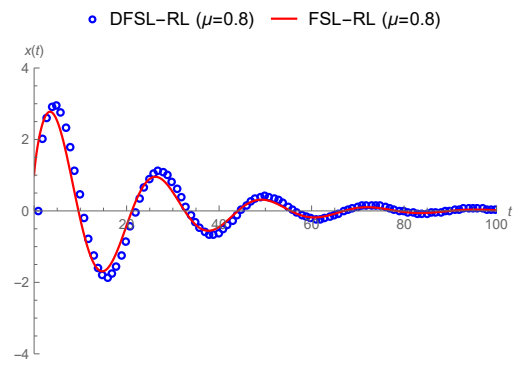

Figure 6.3.26 $\mu=0.8$

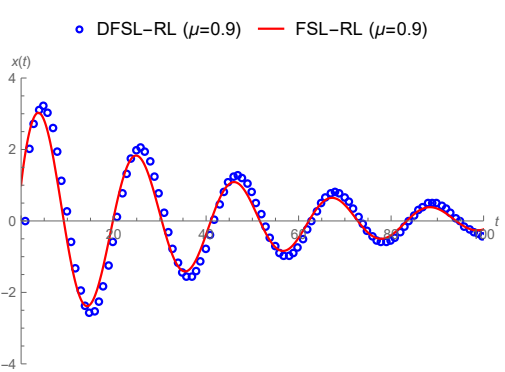

Figure $6.3 .29 \mu=0.9$

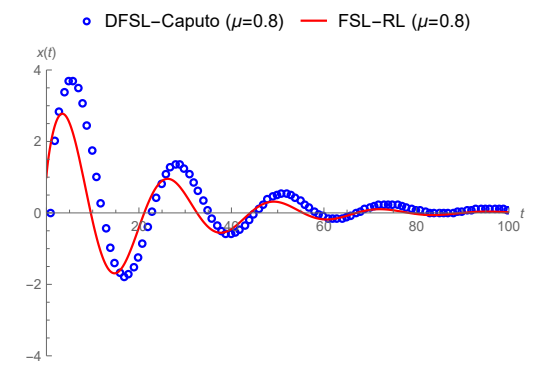

Figure 6.3.27 $\mu=0.8$

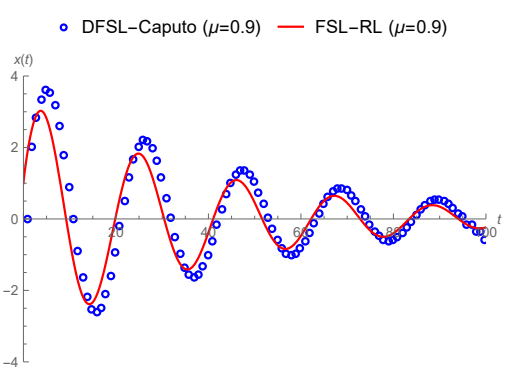

Figure $6.3 .30 \mu=0.9$

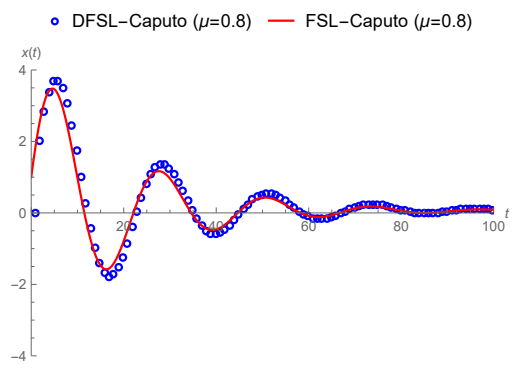

Figure 6.3.28 $\mu=0.8$

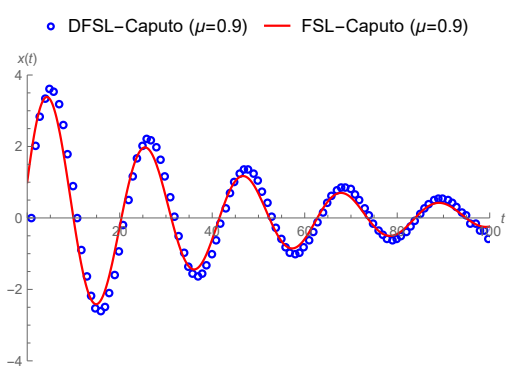

Figure $6.3 .31 \mu=0.9$ 


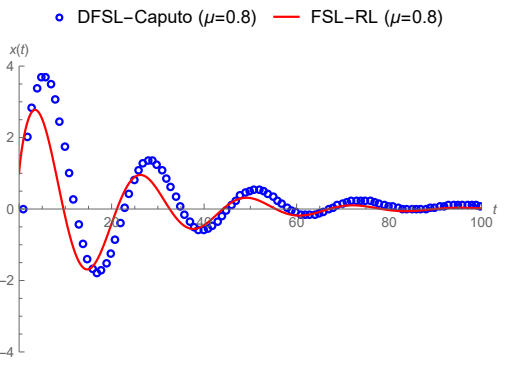

Figure $6.3 .32 \mu=0.8$

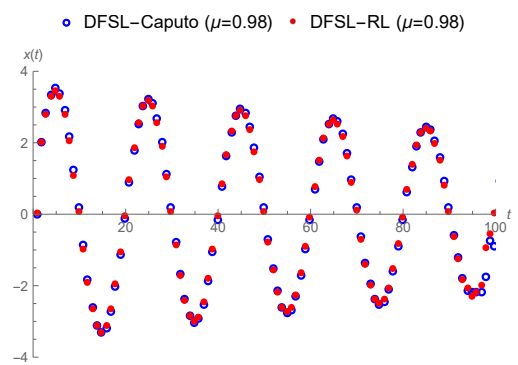

Figure $6.3 .35 \mu=0.98$

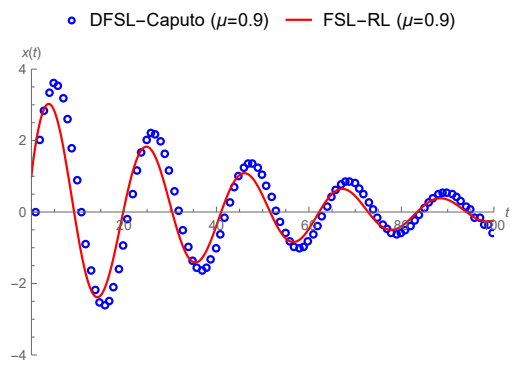

Figure $6.3 .33 \mu=0.9$

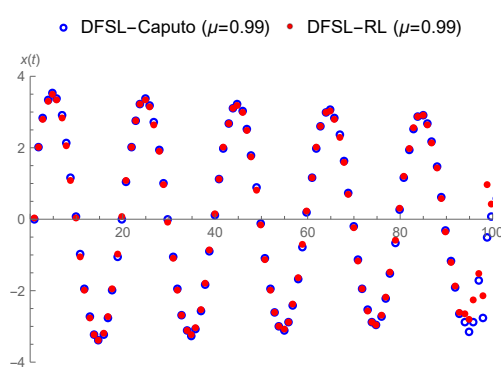

Figure $6.3 .36 \mu=0.99$

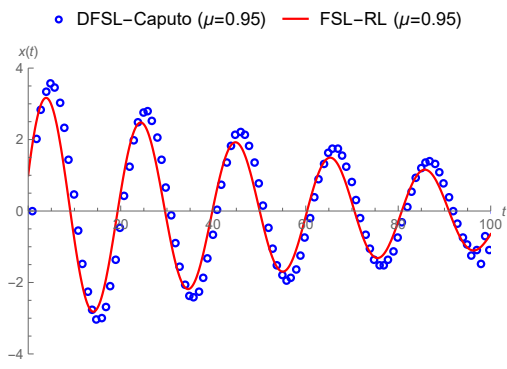

Figure $6.3 .34 \mu=0.95$

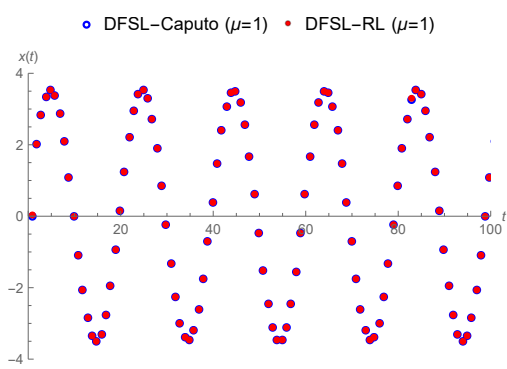

Figure 6.3.37 $\mu=1$

Eigenvalues of DFSL problem (29) - 30) correspond to some specific eigenfunctions for is given with different orders while $q(t)=0$ in Table 6.3.1.

Table 6.3.1 Eigenvalues of the ivp (29-30) under different fractional orders

\begin{tabular}{|c|c|c|c|c|c|c|c|c|}
\hline$\mu$ & $\lambda_{1}$ & $\lambda_{2}$ & $\lambda_{3}$ & $\lambda_{4}$ & $\lambda_{5}$ & $\lambda_{6}$ & $\lambda_{7}$ & $\lambda_{8}$ \\
\hline 0.8 & -0.1029 & -0.077 & -0.068 & -0.047 & -0.037 & -0.023 & -0.014 & -0.0068 \\
\hline 0.85 & -0.1004 & -0.075 & -0.060 & -0.044 & -0.032 & -0.021 & -0.012 & -0.0059 \\
\hline 0.9 & -0.1007 & -0.070 & -0.054 & -0.040 & -0.029 & -0.018 & -0.011 & -0.0051 \\
\hline
\end{tabular}

\begin{tabular}{|c|c|c|c|c|c|c|c|}
\hline$\mu$ & $\lambda_{1}$ & $\lambda_{2}$ & $\lambda_{3}$ & $\lambda_{4}$ & $\lambda_{5}$ & $\lambda_{6}$ & $\lambda_{7}$ \\
\hline 0.9 & -0.0034 & -0.0026 & -0.0019 & -0.0014 & -0.0009 & -0.0005 & -0.00024 \\
\hline 0.94 & -0.0030 & -0.0023 & -0.0017 & -0.0012 & -0.0007 & -0.0004 & -0.00020 \\
\hline 0.98 & -0.0033 & -0.0026 & -0.0020 & -0.0015 & -0.0010 & -0.0006 & -0.00017 \\
\hline
\end{tabular}

\begin{tabular}{|c|c|c|c|c|c|c|c|}
\hline$\mu$ & $\lambda_{1}$ & $\lambda_{2}$ & $\lambda_{3}$ & $\lambda_{4}$ & $\lambda_{5}$ & $\lambda_{6}$ & $\lambda_{7}$ \\
\hline 0.8 & -0.169 & -0.0011 & -0.0007 & -0.00061 & -0.0003 & -0.00024 & -0.00011 \\
\hline 0.85 & -0.256 & -0.0012 & -0.0008 & -0.00065 & -0.0004 & -0.00030 & -0.00018 \\
\hline 0.9 & -0.056 & -0.0010 & -0.0009 & -0.00071 & -0.0005 & -0.00037 & -0.00024 \\
\hline
\end{tabular}

\begin{tabular}{|c|c|c|c|c|c|c|c|}
\hline$\mu$ & $\lambda_{1}$ & $\lambda_{2}$ & $\lambda_{3}$ & $\lambda_{4}$ & $\lambda_{5}$ & $\lambda_{6}$ & $\lambda_{7}$ \\
\hline 0.9 & -0.48 & -0.12 & -0.00019 & -0.00014 & -0.00010 & -0.00006 & -0.000038 \\
\hline 0.94 & -0.40 & -0.18 & -0.00020 & -0.00015 & -0.00011 & -0.00008 & -0.000031 \\
\hline 0.98 & -0.34 & -0.27 & -0.00021 & -0.00017 & -0.00013 & -0.00009 & -0.000024 \\
\hline \multicolumn{70}{c|}{$x(2000)$}
\end{tabular}

Finally, we give the solutions of DFSL problem 29) - 30) with different orders, and potentials in Tables 6.3.2 6.3.4 
Table 6.3.2 $q(t)=0, \lambda=-0.01$

\begin{tabular}{|c|c|c|c|c|c|}
$x(t)$ & $\mu=0.1$ & $\mu=0.2$ & $\mu=0.5$ & $\mu=0.7$ & $\mu=0.9$ \\
$x(0)$ & 2 & 2 & 2 & 2 & 2 \\
$x(1)$ & 2.8 & 2.8 & 2.8 & 2.8 & 2.8 \\
$x(2)$ & 3.5 & 3.48 & 3.42 & 3.38 & 3.34 \\
$x(5)$ & 5.100 & 4.911 & 4.260 & 3.748 & 3.166 \\
$x(7)$ & 5.831 & 5.457 & 4.128 & 3.046 & 1.783 \\
$x(9)$ & 6.357 & 5.763 & 3.645 & 1.933 & -0.033 \\
$x(12)$ & 6.860 & 5.906 & 2.640 & 0.225 & -2.207 \\
$x(15)$ & 7.122 & 5.817 & 1.692 & -0.812 & -2.493 \\
$x(16)$ & 7.172 & 5.757 & 1.437 & -0.948 & -2.131 \\
$x(18)$ & 7.229 & 5.612 & 1.040 & -0.925 & -0.937 \\
$x(20)$ & 7.245 & 5.449 & 0.794 & -0.606 & 0.487
\end{tabular}

Table 6.3.3 $\lambda=-0.01, \mu=0.8$

\begin{tabular}{|c|c|c|c|}
$x(t)$ & $q(t)=1$ & $q(t)=\frac{1}{t+2}$ & $q(t)=\frac{1}{\sqrt{t+2}}$ \\
$x(0)$ & 2 & 2 & 2 \\
$x(1)$ & 2.98 & 2.98 & 2.98 \\
$x(2)$ & 0.954 & 2.940 & 2.213 \\
$x(5)$ & -0.552 & 0.581 & -1.719 \\
$x(7)$ & 1.345 & -0.935 & -1.435 \\
$x(9)$ & -0.644 & -1.745 & 0.261 \\
$x(12)$ & 0.480 & -1.675 & 1.397 \\
$x(15)$ & -0.286 & -0.678 & 0.174 \\
$x(16)$ & -0.363 & -0.271 & -0.287 \\
$x(18)$ & 0.227 & 0.514 & -0.764 \\
$x(20)$ & 0.079 & 1.165 & -0.563
\end{tabular}

Table 6.3.4 $\lambda=-0.01, \mu=0.9$

\begin{tabular}{|c|c|c|c|}
$x(t)$ & $q(t)=1$ & $q(t)=\frac{1}{t+2}$ & $q(t)=\frac{1}{\sqrt{t+2}}$ \\
$x(0)$ & 2 & 2 & 2 \\
$x(1)$ & 2.98 & 2.98 & 2.98 \\
$x(2)$ & 0.952 & 2.938 & 2.211 \\
$x(5)$ & -0.724 & 0.040 & -2.363 \\
$x(7)$ & 2.030 & -1.901 & -1.821 \\
$x(9)$ & -1.227 & -2.851 & 0.719 \\
$x(12)$ & 1.049 & -2.338 & 2.110 \\
$x(15)$ & -0.866 & -0.456 & -0.441 \\
$x(16)$ & -1.112 & 0.245 & -1.255 \\
$x(18)$ & 0.740 & 1.513 & -1.795 \\
$x(20)$ & 0.215 & 2.418 & -0.866
\end{tabular}




\subsection{Discussions on Eigenvalues and Eigenfunctions of DFSL, DSL, FSL, SL Problems}

Now, let us evaluate together the problems (29) - (30) DFSL, (32) - (33) DSL, (35) - (36) FSL and (38) - 39) $S L$ by taking the second initial conditions as zero.

If we consider $\boldsymbol{D F S} \boldsymbol{L}$ problem as follows

$$
\begin{aligned}
& { }^{C} \Delta_{0}^{\mu}(\Delta x(t-\mu))=\lambda x(t), \\
& x(-1)=1, \Delta x(-1)=0,
\end{aligned}
$$

here $t \in \mathbb{N}_{-\mu}^{b-\mu}, b$ is a finite integer, then its analytical solution

$$
x(t)=-E_{(\mu+1,0)}(\lambda, t+1)+E_{(\mu+1,1)}(\lambda, t+1) .
$$

If we consider $\boldsymbol{D S L}$ problem as follows

$$
\begin{gathered}
\Delta^{2} x(t-1)=\lambda x(t), \\
x(-1)=1, \Delta x(-1)=0,
\end{gathered}
$$

here $t \in \mathbb{N}_{-1}^{b-1}, b$ is a finite integer, then its analytical solution

$$
x(t)=\left(\frac{-\sqrt{\lambda}+\sqrt{\lambda+4}}{2 \sqrt{\lambda+4}}\right)\left(\frac{2+\lambda+\sqrt{\lambda(\lambda+4)}}{2}\right)^{t}+\left(\frac{\sqrt{\lambda}+\sqrt{\lambda+4}}{2 \sqrt{\lambda+4}}\right)\left(\frac{2+\lambda-\sqrt{\lambda(\lambda+4)}}{2}\right)^{t} .
$$

If we consider $\boldsymbol{F S} \boldsymbol{L}$ problem as follows

$$
\begin{aligned}
& { }^{C} D_{0^{+}}^{\mu}\left(x^{\prime}(t)\right)=\lambda x(t), \\
& x(0)=1, x^{\prime}(0)=0,
\end{aligned}
$$

$t \in[0, b]$, then its analytical solution

$$
x(t)=E_{\mu+1}\left(\lambda t^{\mu+1}\right) .
$$

If we consider $\boldsymbol{S} \boldsymbol{L}$ problem as follows

$$
\begin{gathered}
x^{\prime \prime}(t)=\lambda x(t), \\
x(0)=1, x^{\prime}(0)=0,
\end{gathered}
$$

$t \in[0, b]$, then its analytical solution

$$
x(t)=\cosh \sqrt{\lambda} t .
$$

Eigenvalues of these problems are the roots of the following boundary condition

$$
x(50)=0 .
$$

Hence, if we apply the solutions of these four problems to the boundary condition above, we can find the eigenvalues of these problems for the orders $\mu=0.9$ and $\mu=0.99$ approximately in Table 6.4.1 and Table 6.4.2.

Eigenfunctions are obtained by writing these eigenvalues into the solution of these four problems. 
Table $6.4 .1 \mu=0.9$

\begin{tabular}{|r|r|r|r|r|r|r|r|r|}
\hline & $\lambda_{1}$ & $\lambda_{2}$ & $\lambda_{3}$ & $\lambda_{4}$ & $\lambda_{5}$ & $\lambda_{6}$ & $\lambda_{7}$ & $\lambda_{8}$ \\
\hline DFSL & -0.292 & -0.231 & -0.177 & -0.129 & -0.088 & -0.055 & -0.029 & $\mathbf{- 0 . 0 0 1}$ \\
\hline FSL & -0.305 & -0.241 & -0.183 & -0.133 & -0.091 & -0.056 & -0.029 & $\mathbf{- 0 . 0 0 1}$ \\
\hline DSL & $\mathbf{- 0 . 0 0 0 9}$ & -0.0087 & -0.024 & -0.047 & -0.077 & -0.115 & -0.161 & -0.273 \\
\hline SL & $\mathbf{- 0 . 0 0 0 9}$ & -0.0039 & -0.0088 & -0.015 & -0.024 & -0.035 & -0.048 & -0.079 \\
\hline
\end{tabular}

Table $6.4 .2 \mu=0.99$

\begin{tabular}{|r|r|r|r|r|r|r|r|r|}
\hline & $\lambda_{1}$ & $\lambda_{2}$ & $\lambda_{3}$ & $\lambda_{4}$ & $\lambda_{5}$ & $\lambda_{6}$ & $\lambda_{7}$ & $\lambda_{8}$ \\
\hline DFSL & -0.340 & -0.274 & -0.215 & -0.117 & -0.078 & -0.047 & -0.024 & $\mathbf{- 0 . 0 0 1}$ \\
\hline FSL & -0.358 & -0.287 & -0.168 & -0.120 & -0.080 & -0.049 & -0.025 & $\mathbf{- 0 . 0 0 1}$ \\
\hline DSL & $\mathbf{- 0 . 0 0 0 9}$ & -0.0087 & -0.024 & -0.047 & -0.077 & -0.115 & -0.161 & -0.273 \\
\hline SL & $\mathbf{- 0 . 0 0 0 9}$ & -0.0039 & -0.0088 & -0.015 & -0.024 & -0.035 & -0.048 & -0.079 \\
\hline
\end{tabular}

\section{Conclusion}

We consider firstly DFSL problem within Caputo and Riemann-Liouville sense, then we find the representation of solutions respectively by means of $\mathcal{Z}$-transformation. Subsequently, we obtain analytical solution of DFSL problem within Caputo and Riemann-Liouville sense by taking $q(t)=0$. Consequently, we observe that while the order $\mu \rightarrow 1$ the solution of DFSL problem (31) approaches to the solution of $D S L$ problem (34) in figure 1 .

We consider secondly the solutions of DFSL, DSL, FSL and SL problems. We observe the solution of DSL and SL problems almost match in any order $\mu$, and we observe the solutions of DFSL and FSL problem almost match in any order $\mu$ in figures 2(a) 2(1). Besides that, we observe that all solutions of DFSL, DSL, FSL and SL problems almost coincide to each other as $\mu=1$ in figures 2(m) 2(o),

Following, we consider DFSL problem in R-L sense and we compare its solutions with the solutions of FSL problem in R-L sense. We observe that solutions of these two problems almost matches in any order $\mu$, however, it is observed that there exists difference between the solutions of DFSL problem in Caputo sense and FSL in R-L sense. Besides that, we compare solutions of DFSL in Caputo sense with the solutions of FSL problem in Caputo sense and we observe that solutions of these two problems almost matches in any order $\mu$. Then, we compare solutions of DFSL problem in R-L and Caputo sense and we observe that solutions coincide while the order $\mu \rightarrow 1$.

Consequently, we observe that all solutions for DFSL R-L-FSL R-L-DFSL Caputo-DFSL CaputoFSL Caputo-DSL-SL matches while the order $\mu=1$. However, we can observe that solutions of DFSL R-L-FSL R-L matches, and solutions of DFSL Caputo-FSL Caputo matches in any order. We conclude that all solutions for DFSL are compatible with the solutions of FSL, DSL and SL problems. A comparison in this style is rather original and there is no known study in delta fractional eigenvalue problems. Thus, this study will attract the attention of readers working in this area.

Moreover, we compare the eigenvalues of the solutions of DFSL problem under different orders in Table 6.3.1. Then, we compare the solutions of DFSL problem with different orders, and different potentials in Tables 6.3.2 6.3.4. However, we discuss on eigenvalues of DFSL problem in the last section in Table 6.4.1 and Table 6.4.2.

\section{Acknowledgment}

This paper includes a part of Ph.D. thesis data of Ramazan OZARSLAN. 


\section{Conflict of interest}

The authors declare no potential conflict of interests.

\section{References}

[1] Rudolf, H., Applications of fractional calculus in physics. World Scientific,2000.

[2] Anatoly, A. K., Hadamard-type fractional calculus, Journal of the Korean Mathematical Society 38.6 (2001): 1191-1204.

[3] Caputo, M., Fabrizio, M., A new definition of fractional derivative without singular kernel, Progress in Fractional Differentiation and Applications,2015 1, 1-13.

[4] Atangana, A., and Baleanu, D., New fractional derivatives with nonlocal and non-singular kernel: theory and application to heat transfer model, Thermal Science,2016 20, 757-763.

[5] Oliveira, D. S., de Oliveira, E. C., Hilfer-Katugampola fractional derivatives. Computational and Applied Mathematics,2018 37, 3672-3690.

[6] Chapman, S., On Non-Integral Orders of Summability of Series and Integrals, Proceedings of the London Mathematical Society,1911 2, 369-409.

[7] Kuttner, B., On differences of fractional order, Proceedings of the London Mathematical Society, 1957 3, 453-466.

[8] Díaz, J. B., Osler, T. J., Differences of fractional order, Mathematics of Computation,1974 28, 185-202.

[9] Granger, C. W., Joyeux, R., An introduction to long-memory time series models and fractional differencing, Journal of time series analysis, 1980 1, 15-29.

[10] Isaacs, G. L., Exponential laws for fractional differences, Mathematics of Computation,1980 35, 933-936.

[11] Hosking, J. R. M., Fractional differencing, Biometrika,1981 68, 165-176.

[12] Miller, K. S., Ross, B. (1988, May). Fractional difference calculus. In Proceedings of the international symposium on univalent functions, fractional calculus and their applications ( $\mathrm{pp}$. 139-152).

[13] Hirota, R., Lectures on difference equations. Science-sha,2000.

[14] Anastassiou, G. A., Discrete fractional calculus and inequalities. arXiv preprint arXiv:0911.3370 (2009).

[15] Anastassiou, G. A. (2010). Nabla discrete fractional calculus and nabla inequalities. Mathematical and Computer Modelling, 51(5-6), 562-571.

[16] Abdeljawad, T., "On Riemann and Caputo fractional differences." Computers \& Mathematics with Applications 62.3 (2011): 1602-1611. 
[17] Abdeljawad, T., Dual identities in fractional difference calculus within Riemann, Advances in Difference Equations 2013.1 (2013): 36.

[18] Abdeljawad, T., On delta and nabla Caputo fractional differences and dual identities, Discrete Dynamics in Nature and Society 2013 (2013).

[19] Miller, K. S., Ross, B., An introduction to the fractional calculus and fractional differential equations, Wiley-Interscience; 1 edition, 1993.

[20] Podlubny, I., Fractional differential equations: an introduction to fractional derivatives, fractional differential equations, to methods of their solution and some of their applications (Vol. 198). Elsevier,1998.

[21] Samko, S. G., Kilbas, A. A., Marichev, O. I., Fractional integrals and derivatives: theory and applications, CRC Press, 1993.

[22] Baleanu, D., \& Fernandez, A., On Fractional Operators and Their Classifications. Mathematics,(2019) 7(9), 830.

[23] Klimek, M., Agrawal, O. P. (2012, May). On a regular fractional Sturm-Liouville problem with derivatives of order in $(0,1)$. In Proceedings of the 13th International Carpathian Control Conference (ICCC) (pp. 284-289). IEEE.

[24] Ciesielski, M., Klimek, M., \& Blaszczyk, T., The fractional Sturm-Liouville problem-Numerical approximation and application in fractional diffusion. Journal of Computational and Applied Mathematics,(2017) 317, 573-588.

[25] Bas, E., Fundamental spectral theory of fractional singular Sturm-Liouville operator, Journal of Function Spaces and Applications,2013 2013.

[26] Bas, E., Metin, F., Fractional singular Sturm-Liouville operator for Coulomb potential, Advances in Difference Equations, 2013 2013, 300.

[27] Dehghan, M., Mingarelli, A. B., Fractional Sturm-Liouville eigenvalue problems, II.2017 arXiv preprint arxiv:1712.09894.

[28] Almeida, R., Malinowska, A., Morgado, M. L., Odzijewicz, T., Variational methods for the solution of fractional discrete/continuous Sturm-Liouville problems, Journal of Mechanics of Materials and Structures 12.1 (2016): 3-21.

[29] Al-Refai, M., Abdeljawad, T., Fundamental results of conformable Sturm-Liouville eigenvalue problems. Complexity, (2017), 2017.

[30] Bas, E., \& Ozarslan, R. (2019). Theory of discrete fractional Sturm-Liouville equations and visual results. AIMS Mathematics, 4(3): 593-612.

[31] Abdeljawad, T., Mert, R., \& Peterson, A., Sturm Liouville equations in the frame of fractional operators with exponential kernels and their discrete versions. Quaestiones Mathematicae (2018): 1-19.

[32] Qureshi, S., Yusuf, A., Modeling chickenpox disease with fractional derivatives: From caputo to atangana-baleanu. Chaos Solitons Fractals, (2019) 122, 111-118. 
[33] Bas, E., Ozarslan, R., Baleanu, D., Ercan, A., Comparative simulations for solutions of fractional Sturm-Liouville problems with non-singular operators. Advances in Difference Equations, 2018(1), (2018), 350.

[34] Atıc1, F. M., Şengül, S., Modeling with fractional difference equations, Journal of Mathematical Analysis and Applications,2010 369, 1-9.

[35] Atıcı F. M., Eloe, P. W., Linear forward fractional difference equations, Communications in Applied Analysis,2015 19, 31-42.

[36] Atıcı F. M., Uyanik, M., Analysis of discrete fractional operators, Applicable Analysis and Discrete Mathematics, 2015 9, 139-149.

[37] Mozyrska, D., Girejko, E., Wyrwas, M., Comparison of h-difference fractional operators, In Advances in the theory and applications of non-integer order systems (pp. 191-197). Springer, Heidelberg,2013.

[38] Mozyrska, D., Wyrwas, M., (2014, June). Solutions of fractional linear difference systems with Caputo-type operator via transform method. In ICFDA'14 International Conference on Fractional Differentiation and Its Applications 2014 (pp. 1-6). IEEE.

[39] Baleanu, D., Rezapour, S., Salehi, S., On some self-adjoint fractional finite difference equations, Journal of Computational Analysis \& Applications, 2015 19, 59-67.

[40] Mohan, J. J., Deekshitulu, G. V. S. R., Fractional order difference equations, International Journal of Differential Equations,2012 2012.

[41] Abdeljawad, Thabet, Fahd Jarad, and Dumitru Baleanu. A semigroup-like property for discrete Mittag-Leffler functions. Advances in Difference Equations 2012.1 (2012): 72.

[42] De Moivre, A., Miscellanes Analytica de Seriebus et Quatratoris, London, 1730.

[43] Laplace, P. S., Theorie Analytique de Probabilities, Part I: Du Calcul des Functions Generatrices, Paris, 1812.

[44] Bohner, M., Peterson, A., 2012. Dynamic equations on time scales: An introduction with applications. Springer Science \& Business Media.

[45] Mittag-Leffler, G. M., Sur la nouvelle fonction $E_{\alpha}(x)$, CR Acad. Sci. Paris, 1903137, 554-558.

[46] Nagai, A., Discrete Mittag-Leffler function and its applications (New Developments in the Research of Integrable Systems: Continuous, Discrete, Ultra-discrete), Kyoto University Institute of Mathematical Analysis Departmental Bulletin Paper,2003 1302, 1-20.

[47] Atici, F. M., Eloe, P. W., A transform method in discrete fractional calculus, International Journal of Difference Equations,2007 2, 165-176.

[48] Holm, M. T., The Laplace transform in discrete fractional calculus, Computers \& Mathematics with Applications,2011 62, 1591-1601.

[49] Abu-Saris, R., Al-Mdallal, Q., On the asymptotic stability of linear system of fractional-order difference equations, ractional Calculus and Applied Analysis 16.3 (2013): 613-629. 
[50] Xiao-yan, L., Wei, J., Solving fractional difference equations using the Laplace transform method, In Abstract and Applied Analysis,2014 2014, Hindawi.

[51] Mozyrska, D., Wyrwas, M., The $\mathcal{Z}$-Transform Method and Delta Type Fractional Difference Operators, Discrete Dynamics in Nature and Society, 20152015.

[52] Wu, G. C., Baleanu, D., Zeng, S. D., Luo, W. H., Mittag-Leffler function for discrete fractional modelling. Journal of King Saud University-Science, 201628, 99-102.

[53] Anh, P.T., Babiarz, A., Czornik, A., Niezabitowski, M., Siegmund, S., Variation of constant formulas for fractional difference equations, Archives of Control Sciences 28 (2018).

[54] Boros, G. and Moll, V., Irresistible Integrals: Symbols, Analysis and Experiments in the Evaluation of Integrals, Cambridge University Press, Cambridge,2004.

[55] Goodrich, C., Peterson, A. C., 2015. Discrete fractional calculus. Berlin: Springer.

[56] Kelley, W. G., Peterson, A. C., Difference equations: an introduction with applications. Academic press, 2001.

[57] Girejko, E., Pawłuszewicz, E., Wyrwas, M., The Z-transform method for sequential fractional difference operators, In Theoretical Developments and Applications of Non-Integer Order Systems2016 (pp. 57-67). Springer, Cham. 